“(C) 2015 IEEE. Personal use of this material is permitted. Permission from IEEE must be obtained for all other uses, in any current or future media, including reprinting/republishing this material for advertising or promotional purposes, creating new collective works, for resale or redistribution to servers or lists, or reuse of any copyrighted component of this work in other works." 


\title{
High-Frequency Magnetic-Link Medium-Voltage Converter for Superconducting Generator-Based High-Power Density Wind Generation Systems
}

\author{
Md. Rabiul Islam, Youguang Guo, Senior Member, IEEE, Jianguo Zhu, Senior Member, IEEE, Haiyan Lu, Member, \\ IEEE, and Jian Xun Jin, Senior Member, IEEE
}

\begin{abstract}
Recent advances in solid-state semiconductors and magnetic materials have provided the impetus for high-frequency magnetic-link-based modular medium-voltage power conversion systems, which would be a possible solution to reduce further the weight and volume of superconducting generator-based wind generation systems. To verify this new concept, in this paper, a laboratory prototype of $5 \mathrm{kVA}$ high-frequency magnetic-link modular power conversion system is developed for a scaled down $1.2 \mathrm{kV}$ grid application. The design and implementation of the prototyping, test platform, and the experimental results are analyzed and discussed. It is expected that the proposed new technology will have great potential for superconducting generator-based wind farm applications.
\end{abstract}

Index Terms-Superconducting generator, modular mediumvoltage converter, high-frequency magnetic-link, high-power density, wind generation system.

\section{INTRODUCTION}

$\mathrm{O}$ FFSHORE wind farms have attracted considerable interest in recent years due to the following facts: (1) offshore winds tend to flow at higher speeds than onshore winds and this allow turbines to produce more electricity at offshore; (2) unlike onshore winds, offshore breezes can be stronger in afternoons, which match the time when load demands are relatively high; and (3) due to the fact that wind farms can cover large areas of land, e.g. a wind farm consisting of $54 \times 3.6 \mathrm{MW}$ turbines could occupy about $20 \mathrm{~km}^{2}$ of land area, offshore wind farms can save land rental expenses, which is equivalent to $10-18 \%$ of the total operating and maintenance costs of a wind farm. According to the statistical data, the global cumulative installed capacity of offshore wind farms in 2008, 2010 and 2012 were $1.50,3.08$ and $5.41 \mathrm{GW}$, respectively, almost doubled in every two years. It is expected that the global offshore installed capacity will increase to approximately $20 \mathrm{GW}$ by 2015 and rise sharply to $104 \mathrm{GW}$ by

Manuscript received May 8, 2014; accepted June 11, 2014. Date of publication; date of current version. This paper was recommended by $\mathrm{H}$. Jia.

M. R. Islam, Y. G. Guo, J. G. Zhu, and H. Y. Lu are with the Faculty of Engineering and Information Technology, University of Technology Sydney, PO Box 123, Broadway, NSW 2007, Australia (e-mail: Md.Islam@uts.edu.au, Rabiulbd@hotmail.com; Youguang.Guo-1@uts.edu.au; Jianguo.Zhu@uts. edu.au; Haiyan.Lu@uts.edu.au).

J. X. Jin is with the Center of Applied Superconductivity and Electrical Engineering, University of Electronic Science and Technology of China, Chengdu, China (e-mail: jxjin@uestc.edu.cn).

Color versions of one or more of the figures in this paper are available online at http://ieeexplore.ieee.org.

Digital Object Identifier 10.1109/TASC.2014.
2025. Therefore, grid integration of large-scale wind farm has attracted a high degree of attention in recent years [1].

Although the power rating of wind turbine generators has been increasing rapidly, the voltage rating of the most common generators is in the range of 380-690 V. Therefore, to integrate the scattered wind turbine generators into a medium-voltage grid (e.g. 11-33 kV) before the voltage stepup for long distance transmission, a power-frequency (i.e. 50 or $60 \mathrm{~Hz}$ ) transformer is commonly used to step-up the voltage. The wind turbine generators are usually heavy and large, especially for offshore applications. For example, a 10 MW permanent magnet direct-drive generator is above $300 \mathrm{t}$ and its diameter is larger than $10 \mathrm{~m} \mathrm{[2].} \mathrm{The} \mathrm{weight} \mathrm{and}$ volume of a $0.69 / 33 \mathrm{kV}, 2.6 \mathrm{MVA}$ transformer are typically in the range of 6-8 $\mathrm{t}$ and 5-9 $\mathrm{m}^{3}$, respectively. A liquid-filled 2 MVA step-up transformer uses about $900 \mathrm{~kg}$ of liquid as the coolant and insulator, which requires regular monitoring and replacement. Moreover, insulation materials of transformers introduce environment concerns. For example, according to European requirements, more than $65 \%$ of the liquid must be degraded in 28 days. In an offshore wind farm, the generator and transformer are usually installed at a height of about 80 meters inside the nacelle. The heavy and large generator and step-up-transformer significantly increase the weight and volume of the nacelle as well as increase the mechanical stress of the tower. These drawbacks are critical in offshore applications, where the costs of installation and regular maintenance are extremely high. For example, the foundation size of a $2.3 \mathrm{MW}$ wind turbine is $314 \mathrm{~m}^{2}$ and the approximate weight is $2000 \mathrm{t}$ or more. The installation cost of an offshore wind farm takes up, on average, approximately $20 \%$ of the capital cost. Typically, the maintenance cost of an offshore wind farm is about 2.3 cents per kilowatt-hour. Recently, considerable attention has been directed towards reducing the weight and size by using superconducting generators and eliminating step-up-transformers.

\section{RESEARCH AND DEVELOPMENT}

\section{A. Superconducting generators}

Superconductors are materials which carry almost loss-free electrical currents, when cooled down below their critical temperatures [3]. From 1975 to 1979, a detailed conceptual design study of 300 and 1200 MVA steam turbine generators 
utilizing superconducting field windings was carried out [4]. The high temperature superconductor (HTS) materials were discovered in 1986 by International Business Machines (IBM) researchers and then gradually improved with a concomitant reduction in cost. Compared with copper conductor, the HTS features very low losses and high current densities [5]. Therefore, a superconductor based compact and lightweight generator may reduce the weight of wind turbine power generation systems. The projected weight of a 10 MW HTS generator is approximately $150-180 \mathrm{t}$, which is roughly only $50 \%$ of that of a permanent magnet direct-drive generator [6]. There are several successful tests of the first generation models and the prototypes of motors and generators using HTS [7], [8]. In 2005, Siemens developed rotors using superconducting field windings. American Superconductor Corporation (AMSC), in collaboration with NorthropGrumman, built the world's highest torque HTS motor for the US Navy at 36.5 MW using bismuth based superconductor bismuth strontium calcium copper oxide (BSCCO) wire. This was delivered in 2007. Recently, Sway Turbine and Windtec Solutions have been developing a $10 \mathrm{MW}$ wind turbine generator, which is called the SeaTitan and this is considered to be the world's most powerful turbine. The SeaTitan wind turbine design employs an HTS generator, which is significantly smaller and lighter and expected to be commercially available by 2015 [9]. Therefore, although it is hopeful to significantly reduce the weight and volume of wind generation systems by using HTS generators, they are still heavy and large especially for offshore applications due to the use of heavy and large-size step-up-transformers and line filters.

\section{B. Step-up-transformer-less direct grid connection}

In order to reduce the volume and weight of wind generation systems, elimination of the step-up-transformer and line filter has attracted significant attention in recent years. The modular multilevel cascaded (MMC) converter topology may be the natural choice for the development of multilevel medium-voltage converters [10]. A multi-coil modular permanent magnet generator [11] and a few 6-phase generators [12] were proposed to eliminate the step-uptransformer and line filter of the wind turbine power generation systems. However, these approaches require special modular generators or multiple traditional generators to generate isolated multiple dc supplies for the $\mathrm{MMC}$ converter, and introduce electrical isolation problem between generator and grid. Recently amorphous alloy-based highfrequency common magnetic-links were proposed to generate isolated and balanced multiple dc supplies for the MMC converter [13], [14]. High-frequency common magnetic-link (instead of common DC-link) based $1 \mathrm{kV}$ converters were successfully validated for wind and solar PV power generation systems [15], [16]. Although the common magnetic-link may ensure equal voltages at the secondary terminals, the topology diminishes modularity of the power conversion system, which is very important for medium or high-voltage high-power system. The implementation of high-power high-frequency inverter is the main challenging issue. Moreover, the leakage inductance may also limit the power capacity of the highfrequency magnetic-link.

In this paper, a new medium-voltage converter is proposed with multiple-cascaded modules for HTS generator-based offshore wind farms, which has the following new features compared with the existing similar systems: (1) no requirement for special or multiple generators; (2) an inherent voltage balance; (3) completely modular in construction; (4) possibility to use commercially available low-cost mature devices; (5) no power limitation; (6) an overall compact and lightweight system; and (7) inherent minimization of the grid isolation problem.

\section{New High-Power DENSITY Wind GeNERATION SyStem}

In this section, we present the new medium-voltage converter with multiple high-frequency magnetic-links for superconducting generator-based offshore wind farms, which is completely modular in construction. Fig. 1 shows the circuit diagram of the medium-voltage power conversion system, where the available rectified wind power (from HTS generator followed by rectifier circuit) is supplied to a few cascaded modules. Each module consists of a high-frequency inverter, a four-winding magnetic-link, and three H-bridge inverters with high-frequency rectifiers. High-frequency inverter converts the rectified wind power to a high-frequency ac, which energies the primary coil of the magnetic-link. To ensure a fixed grid voltage, a constant output voltage of the inverter is maintained. Each secondary winding is connected with an $\mathrm{H}$-bridge cell through a bridge rectifier; each $\mathrm{H}$-bridge inverter cell contributes two voltage steps to the output. The grid electrical isolation and voltage unbalance problems are solved through the high-frequency magnetic-links.

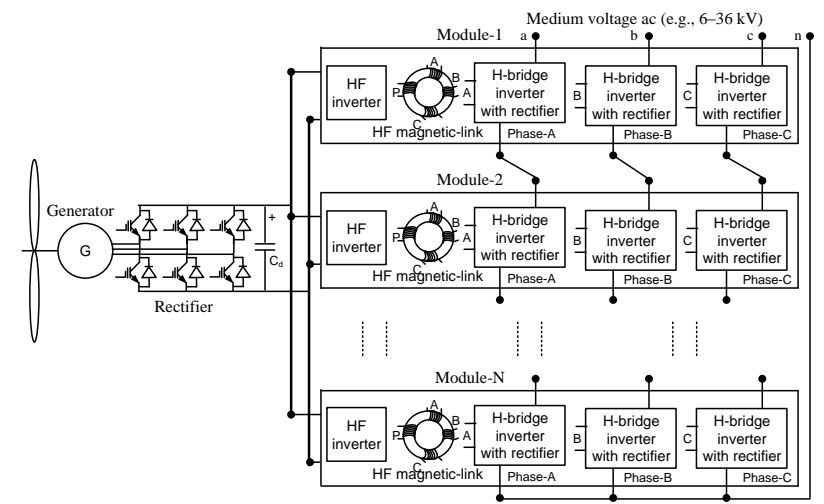

Fig. 1. Circuit diagram of the high-power density wind generation system.
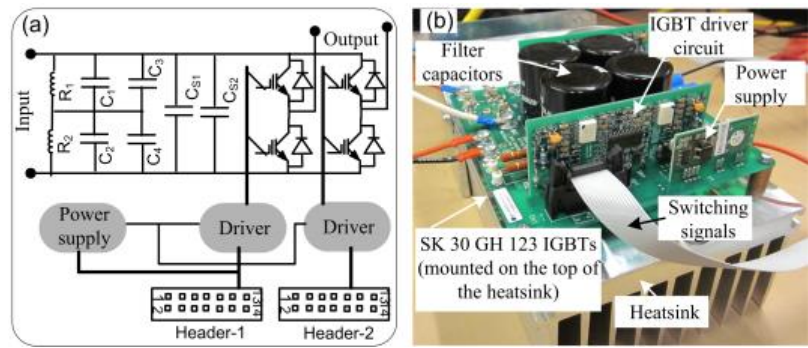

Fig. 2. High-frequency inverter: (a) basic circuit diagram, and (b) photograph of the prototype inverter. 


\section{Design OF The Proposed POWER CONVERSION SYSTEM}

\section{A. High-frequency inverter}

Full-bridge high-frequency inverters are developed by using Semikron SK30GH123 compact insulated gate bipolar transistor (IGBT) modules to generate high-frequency square wave primary excitation voltage of the high-frequency magnetic-links. Fig. 2(a) shows the circuit diagram of the highfrequency inverter. Fig. 2(b) shows the photograph of the prototype inverter. High-speed PWM controller UC3825BN is used to generate the switching signals. The oscillator of the UC3825BN is a saw-tooth which utilizes two pins; one for the timing resistor $R_{T}$ and the other for timing capacitor $C_{T}$. Based on the desired maximum duty cycle, $D_{\max }$ the timing resistor can be calculated from

$$
R_{T}=\frac{3}{10 \times 10^{-3}\left(1-D_{\max }\right)}
$$

Based on the calculated value of timing resistor and desired maximum duty cycle, the timing capacitor can be calculated from

$$
C_{T}=\frac{1.6 \times D_{\max }}{R_{t} \times f} \quad(\mathrm{~F}) .
$$

The gate pulses were measured and compared with the simulation results. The measured pulses were found highly consistent with the simulation results.

\section{B. High-frequency magnetic-links}

In high-frequency magnetic-link design, the winding electromotive force (emf) is proportional to the number of turns, frequency, and magnetic flux linking the winding. For a given power capacity, as the operating frequency increases, the required cross sectional area of magnetic core and the number of turns of the primary and secondary windings can be dramatically reduced. On the other hand, the current density within the conductors can change significantly as the excitation frequency increases because of two main factors, skin effect and proximity effect. Fig. 3(a) shows the skin effect loss in a round conductor for different penetration ratios $(d / \delta)$. The proximity effect can be classified into two types, internal proximity effect and external proximity effect. Fig. 3(b) shows the power loss in a round conductor due to proximity effect. For a small skin depth and proximity effect, the number of layers as well as the conductor diameter should be kept as small as possible. Moreover, the insulated strands should be twisted or braided together to equalize the flux linkages throughout the conductors. To achieve this so as to reduce the winding loss, a Litz wire with small number of layers should be always used in a high-frequency link.
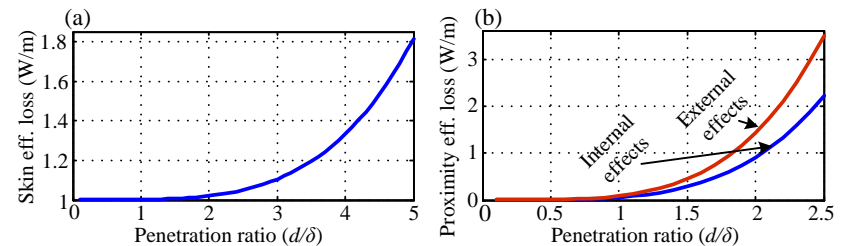

Fig. 3. Power losses due to: (a) skin effect, and (b) proximity effect.

The amorphous alloys have excellent magnetic characteristics for high-frequency applications, such as high permeability, high saturation flux density and relatively low core losses. Of the other amorphous alloys, 2605SA1, $2605 \mathrm{~S} 3 \mathrm{~A}$, and 2714A, the last one has the lowest specific core loss but its saturation flux density is only $0.57 \mathrm{~T}$ [17], [18]. Taking into account the flux density, specific core loss, cost, and availability, the Metglas 2605SA1 stripe of $20 \mu \mathrm{m}$ thickness and $25 \mathrm{~mm}$ width has been chosen as the core material. The other parameters include mass density of 7.18 $\mathrm{g} / \mathrm{cm}^{3}$, saturation flux density of $1.56 \mathrm{~T}$, and specific core loss of $180 \mathrm{~W} / \mathrm{kg}$ at $10 \mathrm{kHz}$ sinusoidal excitation of $1 \mathrm{~T}$. The core losses in the datasheet for this material are experimental results under sinusoidal voltage excitation, where $f$ is the frequency in $\mathrm{kHz}$ and $B$ the magnitude of flux density in T. Therefore, for this design new coefficients are obtained by measurements under square voltage excitation. The formula and newly derived coefficients are presented in (3).

$$
P_{\text {core_square }}=6.26 f^{1.58} B^{1.62}(\mathrm{~W}) \text {. }
$$

If $f$ is the frequency of the excitation voltage, $B_{m}$ is the maximum flux density and $A$ is the cross-section area of the core, then the number of turns can be calculated from

$$
N=\frac{V_{r m s}}{4 f A B_{m}} .
$$

At the beginning, according to power inverter rating, the high-frequency magnetic-link specifications, such as the rated power, frequency, excitation current waveform, and voltage, are calculated. From the specifications of magnetic-link and data sheets of core materials, magnetic-link initial parameters are calculated. These parameters are used as initial values of the optimization process. For structural simplicity, a toroidal structure core is considered. Different factors are considered during the selection of core dimensions, such as the winding dimensions, hole reserve for natural cooling, maximum temperature limits, maximum power loss, availability of core material stripe dimensions, leakage inductance, and possibility to induce equal voltage in multiple secondary windings. Therefore, the design process involves multi-physics problems with some critical decision making tasks.

Scaled down prototype of $2.5 \mathrm{kVA}$ high-frequency magnetic-links with single primary and three secondary windings are developed, as shown in Fig. 4 to generate the balanced isolated three dc supplies for three H-bridge inverter cells.

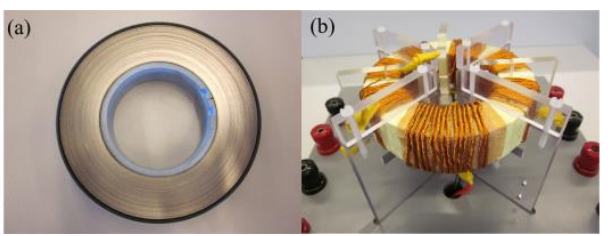

Fig. 4. Photograph of the: (a) core with frame, and (b) magnetic-link.

The magnetic field intensity and magnetic flux density are calculated by measuring the primary coil excitation current and the open circuit terminal voltage of one of the six secondary coils. As the coils are uniformly wound on the toroidal core, the magnetic field intensity $H$ and magnetic flux 
density $B$ within the core can be considered as uniform. By using Ampere's law, the field intensity can be calculated from

$$
H=\frac{N_{1} i(t)}{l_{e}} \quad(\mathrm{~A} / \mathrm{m}),
$$

where $N_{l}$ is the number of turns in the primary coil, $i(t)$ the excitation current, and $l_{e}$ the mean length of the core. By using Faraday's law, the magnetic flux density in the core can be calculated from

$$
B=\frac{1}{N_{2} A_{e}} \int V_{L} d t \quad(\mathrm{~T}),
$$

where $N_{2}$ is number of turns in the pick-up coil and $A_{e}$ the cross sectional area of the core, and $V_{L}$ the pick-up coil voltage. The $B-H$ loops and specific core losses of the highfrequency magnetic-link were analyzed at different excitation frequencies. The plotted $B-H$ loops have been compared with the material manufacturer's data and found highly consistent.

\section{High-frequency rectifiers}

For simplicity, single phase diode rectifiers are considered to convert medium frequency alternating quantity to $\mathrm{dc}$ quantity with fast recovery diodes. Each module is associated with a separate rectifier, such that in total six rectifiers are required for a 3-phase 5-level converter. Fig. 5(a) shows the circuit diagram of the rectifiers. The IXYS DSEE15-12CC super fast recovery $(25 \mathrm{~ns})$ dual diode module is considered for the development of module rectifier of medium voltage converter. Fig. 5(b) shows the photograph of the prototype rectifiers with heat sinks. The dc-link voltages were measured and found approximately equal at about $367 \mathrm{~V}$.

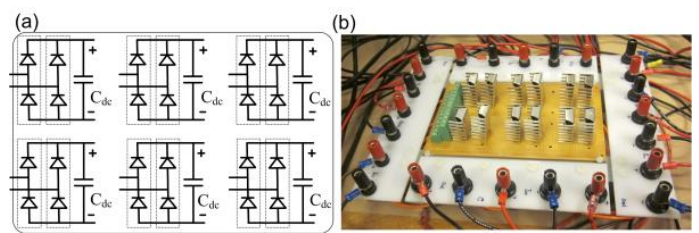

Fig. 5. High-frequency rectifier: (a) circuit, and (b) photograph of the prototype rectifiers.

If $I_{d c}$ is the output rectifier average current, $f_{s}$ is the source voltage frequency, $V_{d c}$ is the output rectifier dc output voltage, $D_{\text {off }}$ is the diode off time or capacitor discharge time factor, and $\gamma$ is the ripple factor, then the minimum value of the capacitor can be calculated from

$$
C=\frac{I_{d c} D_{o f f}}{4 \sqrt{3} \gamma f_{s} V_{d c}} \quad(\mathrm{~F}) .
$$

The 3188EE152T400APA1 aluminum electrolytic capacitor is used for the module rectifier circuit. The heat-sink thermal resistance (heat sink to ambient) can be calculated from

$$
\theta_{S A}=\frac{T_{J}-T_{A}}{P_{D}}-\left(\theta_{J C}+\theta_{C S}\right) \quad\left({ }^{\circ} \mathrm{C} / \mathrm{W}\right),
$$

were $T_{J}$ is the junction temperature, $T_{A}$ is the ambient (air) temperature, $\theta_{J C}$ is the transistor thermal resistance (junction to case), $\theta_{C S}$ is the insulator thermal resistance (case to heat sink), and $P_{D}$ is the power dissipation.

\section{H-bridge inverter cells}

A scaled down $1.2 \mathrm{kV}$ 3-phase 5-level converter is developed, where each phase-leg consists of two H-bridge inverter cells, i.e. two modules in the power conversion system. The dc-link voltage of each $\mathrm{H}$-bridge inverter cell is calculated as $367 \mathrm{~V}$. Considering the market availability, cost and suitability for prototyping, the Semikron compact IGBT modules SK30GH123 have been collected for the development of the H-bridge inverter cells. The module consists of four $\mathrm{N}$-channel $1.2 \mathrm{kV}$ IGBTs in H-bridge connection. The Semikron driver SKHI 20opA is a dual driver for the half-bridge inverter cell, which may drive the IGBT with commutation voltage up to $1.2 \mathrm{kV}$. The Semikron dual IGBT driver isolated power supply SKHI PS1 has been considered. It has the capability to supply energy for up to seven IGBTs with additional transformers. The IGBT module and the driver and protection circuit (with power supply and filtering capacitors of the respective rectifier circuit) are mounted on the top of the heatsink. A field programmable gate array (FPGA)-based control circuit is used to control the magnitude and phase angle, and to ensure the power quality and stability of the converter. The Semikron driver SKHI 20op requires $15 \mathrm{~V}$ gate pulses. The generated gate pulses by Xilinx FPGA XC3S500E are $3.3 \mathrm{~V}$ and these require stepping-up before feeding to the driver SKHI 20op. Therefore, an extra driver circuit has been designed, as shown in Fig. 6 to interface the FPGA and driver SKHI 20op. The Microchip dual high-speed power metal oxide field effect transistor (MOSFET) driver TC4427A is used to design extra driver circuit. Fig. 7 shows a photograph of the prototype MMC converter.

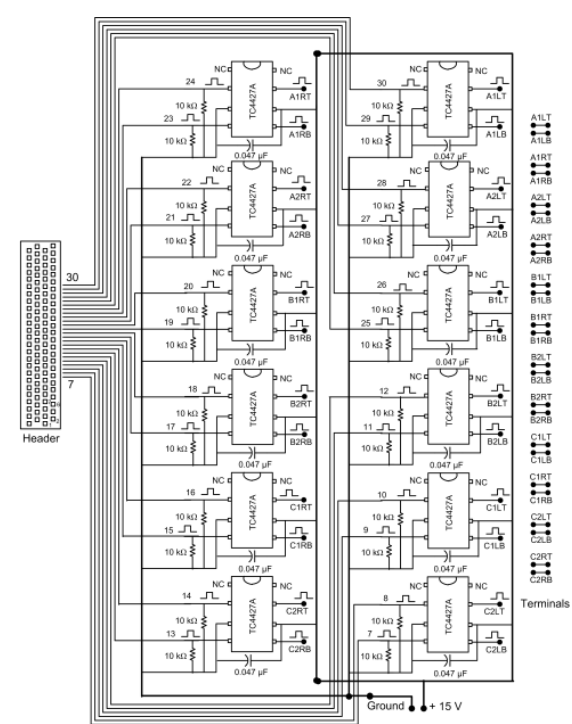

Fig. 6. IC TC4427A-based extra driver circuit for 3-phase 5-level inverter.

\section{EXPERIMENTAL TESTING AND RESULTS ANALYSIS}

The phase-shifted carrier and third harmonic injected sinusoidal reference based pulse width modulation scheme is used in the simulation and experiment. The line-to-line voltages before the filter circuit are measured and they were 
found highly consistent with the theoretical and simulation results. The line-to-line voltages before filter circuit are illustrated in Fig. 8(a). Fig. 8(b) depicts the measured line voltage waveforms after the filter. The line voltage waveforms before filter circuit contains about $19 \%$ total harmonic distortion (THD), and after the filter, it is reduced to less than $2.8 \%$ and they were found highly consistent with the theoretical and simulation results. Fig. 9(a) shows the simulated line voltages of the proposed system after line filter. Fig. 9(b) plots the measured line current waveforms with the filter circuit. As measured, with the filter circuit, the line current waveform contains about $2.72 \%$ THD.

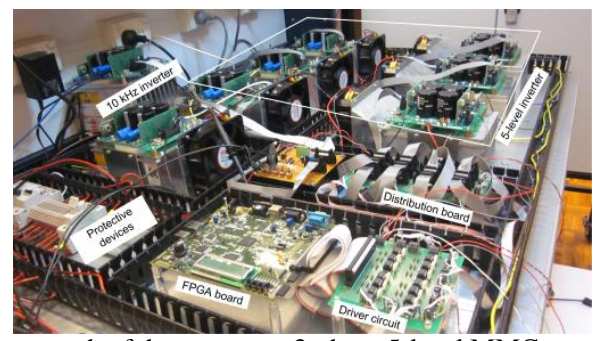

Fig. 7. A photograph of the prototype 3-phase 5-level MMC converter.
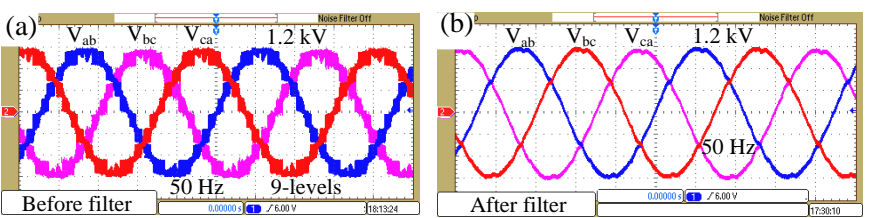

Fig. 8. Measured line-to-line voltages of the prototype system: (a) before line filter circuit, and (b) after line filter circuit.
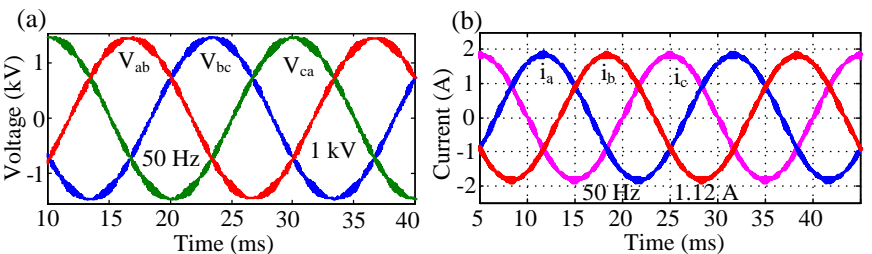

Fig. 9. (a) Simulated line-to-line voltages of the proposed system, and (b) measured line currents of the prototype $1.2 \mathrm{kV}$ power converter.

Because of the significant power losses in the $10 \mathrm{kHz}$ inverter, high-frequency magnetic-link, and fast recovery rectifiers, the full load overall efficiency of the proposed system is only $74 \%$, which is in general about $15 \%$ lower than that of traditional system. The traditional system, however, contains a step-up-transformer and a harmonic neutralizing filter, which together produce about $50 \%$ of the total losses and occupy up to $40 \%$ of the system volume [19]. Therefore, the overall efficiencies of the proposed and conventional systems are similar.

\section{CONCLUSION}

A new high-power density wind generation system is proposed for HTS generator based offshore wind farms. The proposed architecture leads to a compact and lightweight design, which may save large amount of installation, running and maintenance costs of offshore wind power generation systems. The proposed system has been validated by a scaled down $1.2 \mathrm{kV}$ prototype system. The same concept can be used to develop the converter for $6-36 \mathrm{kV}$ system by changing the number of modules.

\section{REFERENCES}

[1] M. WeiWei, J. Hongjie, W. Dan, et al., "Active power regulation of wind power systems through demand response," Science China ETechnological Sciences, vol. 55, no. 6, pp. 1667-1676, Jun. 2012.

[2] R. Qu, Y. Liu, and J. Wang, "Review of supperconducting generator topologies for direct-drive wind turbines," IEEE Trans. Appl. Supercond., vol. 23, no. 3, p. 5201108, Jun. 2013.

[3] L. Xiao, S. Dai, L. Lin, et al., "Development of the world's first HTS power substation," IEEE Trans. Appl. Supercond., vol. 22, no. 3, art. 5000104, Jun. 2012.

[4] S. Minnich, T. Keim, M. Chari, B. Gamble, M. Jefferies, D. Jones, E. Laskaris, and P. Rios, "Design studies of superconducting generators," IEEE Trans. Magn., vol. 15, no. 1, pp. 703-710, Jan. 1979.

[5] L. Xiao, S. Dai, L. Lin, et al., "Development of a $10 \mathrm{kA}$ HTS de power cable," IEEE Trans. Appl. Supercond., vol. 22, no. 3, art. 5800404, Jun. 2012.

[6] H. Li, Z. Chen, and H. Polinder, "Optimization of multibrid permanentmagnet wind generator systems," IEEE Trans. Energy Con., vol. 24, no. 1, pp. 82-92, Mar. 2009.

[7] D. Aized, B. B. Gamble, A. Sidi-Yekhlef, J. P. Voccio, D. I. Driscoll, B. A. Shoykhet, and B. X. Zhang, "Status of the 1000 HP HTS motor development," IEEE Trans. Appl. Supercond., vol. 9, no. 2, pp. 11971200, Jun. 1999.

[8] M. Frank, J. Frauenhofer, P. Hasselt, W. Nick, H. W. Neumueller, and G. Nerowski, "Long-term operational experience with first Siemens 400 kW HTS machine in diverse configurations," IEEE Trans. Appl. Supercond., vol. 13, no. 2, pp. 2120-2123, Jun. 2003.

[9] G. Snitchler, B. Gamble, C. King, and P. Winn, "10 MW class superconductor wind turbine generators," IEEE Trans. Appl. Supercond., vol. 21, no. 3, pp. 1089-1092, Jun. 2011.

[10] M. R. Islam, Y. G. Guo, and J. G. Zhu, "A review of offshore wind turbine nacelle: Technical challenges, and research and development trends," Renew. Sustain. Energy Reviews, vol. 33, pp. 161-176, May 2014

[11] X. Yuan, J. Chai, and Y. Li, "A transformer-less high-power converter for large permanent magnet wind generator systems," IEEE Trans. Sust. Energy, vol. 3, no. 3, pp. 318-329, July 2012.

[12] F. Deng and Z. Chen, "A new structure based on cascaded multilevel converter for variable speed wind turbine," in Proc. $36^{\text {th }}$ Annual Conf. on IEEE Ind. Electron. Society, AZ, USA, pp. 3167-3172, Nov. 2010.

[13] M. R. Islam, Y. G. Guo, and J. G. Zhu, "A medium-frequency transformer with multiple secondary windings for medium-voltage converter based wind turbine generating systems," J. Appl. Phys., vol. 113, no. 17, pp. 17A324, May 2013.

[14] M. R. Islam, Y. G. Guo, Z. W. Lin, and J. G. Zhu, "An amorphous alloy core medium frequency magnetic-link for medium voltage photovoltaic inverters," J. Appl. Phys., vol. 115, no. 17, pp. 17E710, May 2014.

[15] M. R. Islam Y. G. Guo, and J. G. Zhu, "A high-frequency link multilevel cascaded medium-voltage converter for direct grid integration of renewable energy systems," IEEE Trans. Power Electron., vol. 29, no. 8, pp. 4167-4182, Aug. 2014

[16] M. R. Islam, Y. G. Guo, and J. G. Zhu, "A multilevel medium-voltage inverter for step-up-transformer-less grid connection of photovoltaic power plants," IEEE J. Photovoltaics, vol. 4, no. 3, pp. 881-889, May 2014

[17] E. Agheb, M. A. Bahmani, H. K. Hoidalen, and T. Thiringer, "Core loss behavior in high frequency high power transformers-II: Arbitrary excitation," J. Rene. Sust. Energy, vol. 4, no. 3, pp. 033113-1-03311311, Jun. 2012.

[18] R. Burdt, R. C. Curry, K. F. McDonald, P. Melcher, R. Ness, and C. Huang, "Evaluation of nanocrystalline materials, amorphous metal alloy, and ferrites for magnetic pulse compression applications," J. Appl. Phys., vol. 99, no. 8, pp. 08D911-1-08D911-3, Apr. 2006.

[19] F. Z. Peng, J. S. Lai, J. McKeever and J. VanCoevering, "A multilevel voltage source inverter with separate dc sources for static VAR generation," IEEE Trans. Ind. Appl., vol. 32, no. 5, pp. 1130-1138, Sep./Oct. 1996. 